\title{
Localized giant pseudo-polyposis in ulcerative colitis
}

\author{
D. R. FOSTER \\ D.M.R.D., F.R.C.R.
}

Department of Radiology, Royal Perth Hospital, Perth, Western Australia

\begin{abstract}
Summary
Localized giant pseudo-polyposis is a rare complication of ulcerative or granulomatous colitis. A case illustrating the typical radiological findings is described. Recognition of this condition is important since it may simulate malignancy.

\section{Introduction}

The occurrence of pseudo-polyps in ulcerative colitis is a common finding. The reported incidence varies from 12.5 to $74 \%$ (de Dombal et al., 1966; Edwards and Truelove, 1964; Jalan et al., 1969;
\end{abstract}

Bockus et al., 1958) depending on the diagnostic criteria and patient groups studied. Pseudo-polyps represent discrete areas of mucosal inflammation and regeneration and are more commonly seen in the presence of extensive colonic involvement with colitis. They are typically short in height, measuring less than $1.5 \mathrm{~cm}$, and produce no specific symptoms. The majority of current evidence suggests that pseudopolyposis alone is not a pre-malignant feature (Morson and Pang, 1967). They have also been noted to occur in Crohn's colitis.

The following case report illustrates an interesting and rare feature of pseudo-polyposis. Localized giant

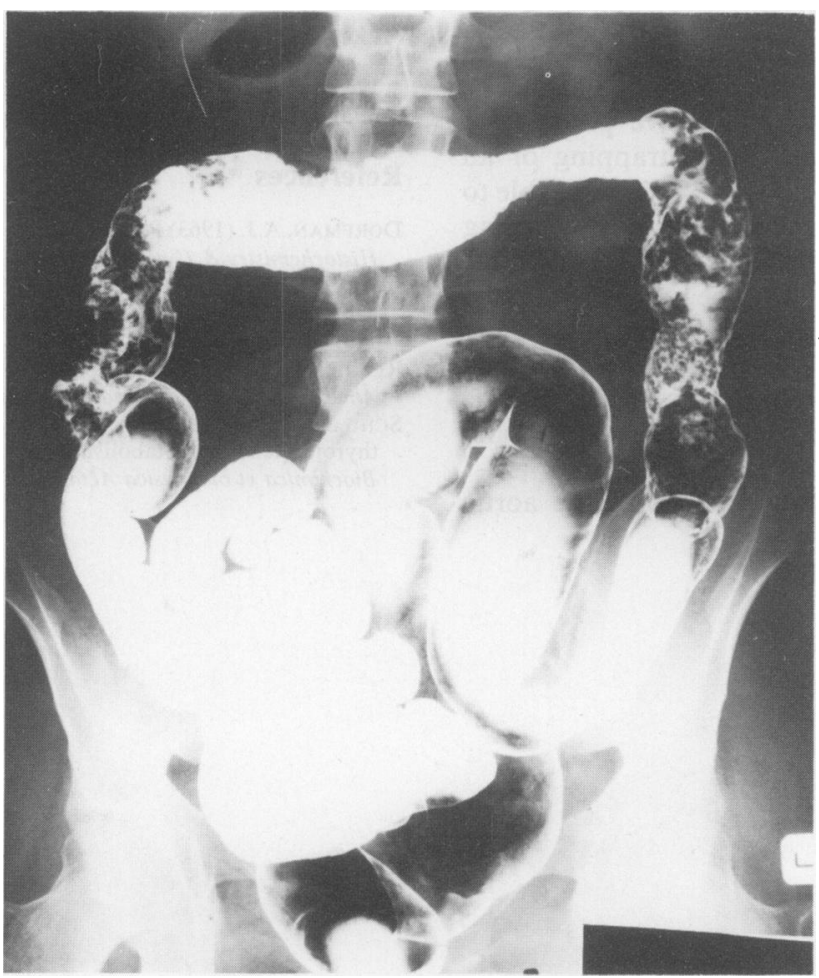

FIG. 1. Multiple colonic pseudo-polyps. 
Pathological examination of the colon (Fig. 3)

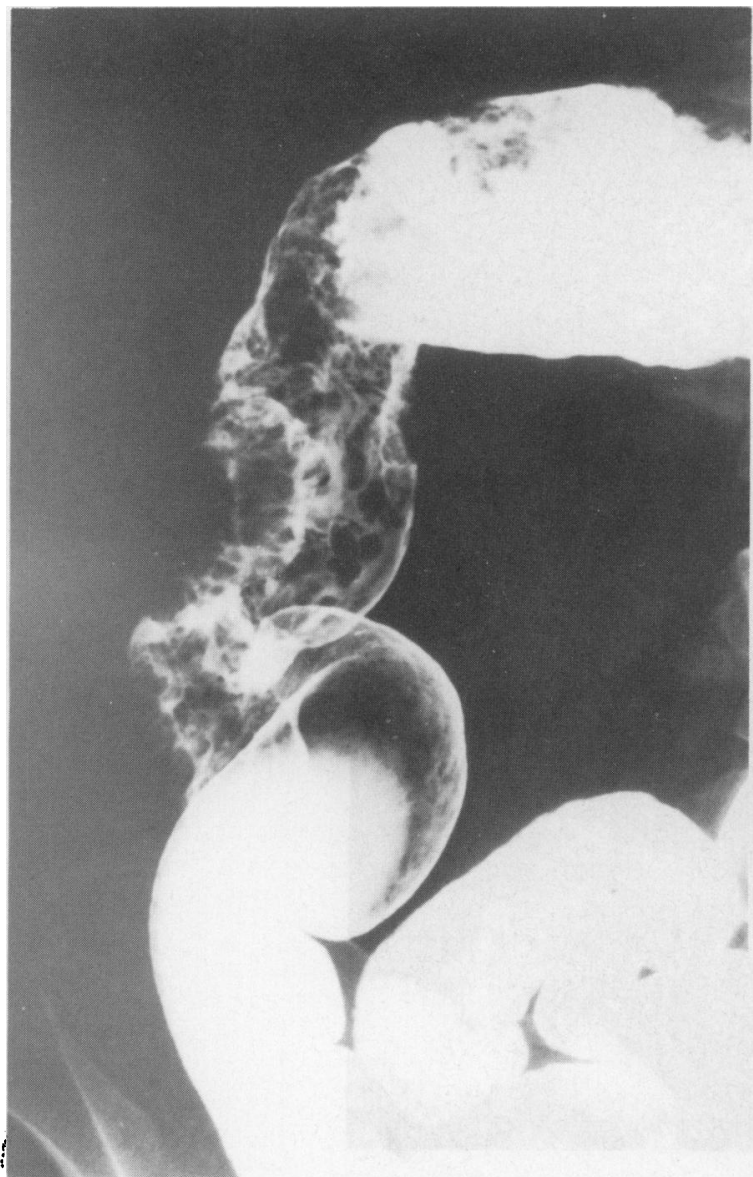

FIG. 2. Localized mass of pseudo-polyps in ascending colon.

pseudo-polyposis represents a collection of pseudopolyps which appear as a large intraluminal colonic mass. This lesion can mimic a carcinoma or villous adenoma on radiological examination.

\section{Case report}

A 30-year-old female patient with a 7 year history of ulcerative colitis was referred for a routine followup barium enema examination.

Multiple pseudo-polyps were seen in the ascending, transverse and descending colon (Fig. 1). In the ascending colon there was a localized mass with a frond-like appearance arising from the lateral wall of the colon (Fig. 2). Further assessment by colonoscopy was unsuccessful and, in view of the possibility of malignancy, total colectomy with ileo-rectal anastomosis was performed, the rectum having a normal appearance on sigmoidoscopy. showed features of long-standing ulcerative colitis. Multiple pseudo-polyps were present together with a large localized mass of pseudo-polyps in the ascending colon. No evidence of a carcinoma was found although dysplastic changes were evident.

\section{Discussion}

Localized giant pseudo-polyposis in ulcerative and granulomatous colitis was reviewed by Joffe (1977). He described 4 new cases and noted a total of 8 previously recorded in the literature. In these 12 patients, the primary disease was ulcerative colitis in 6 patients, granulomatous colitis in 4 patients, whilst in 2 cases the aetiology was uncertain.

Clinical symptoms in patients with localized giant pseudo-polyposis are usually related to the underlying colitis. Discovery of the lesion on a routine follow-up barium enema in a patient with longstanding ulcerative colitis has now occurred in 2 cases. Physical examination is also usually unhelpful although occasionally a palpable mass may be evident (Ferrucci and Vickery 1972; Keating and Mindell 1976; Wills and Han, 1977).

On barium enema examination, localized giant pseudo-polyposis appears as a large mass projecting into the lumen of the colon with numerous frond-like projections arising from the lesion. Multiple pseudopolyps are usually seen in the adjacent colon. Keating and Mindell (1976) noted a lesion causing obstruction but this is very unusual. Localized giant pseudo-polyposis may occur at any site in the colon although it has not been recorded in the rectum.

The important radiological differential diagnosis is, of course, a carcinoma, although carcinoma in ulcerative colitis usually presents as a constricting ulcerative lesion. Although villous adenoma is not a known complication of ulcerative colitis, the radiological appearances of localized giant pseudo-polyposis and villous adenoma may be very similar. Frond-like projections are, however, usually seen in rectal villous adenomas and localized giant pseudo-polyposis has not yet been described in the latter site. Colonic lymphoma may also present as an intraluminal polypoidal mass and should also be considered in differential diagnosis. In the majority of cases, further assessment by colonoscopy and biopsy will be required.

In summary, the diagnosis of localized giant pseudo-polyposis can be suggested on barium enema examination. The typical appearances of an intraluminal mass lesion with frond-like projections, often with numerous pseudo-polyps elsewhere in the colon, can enable recognition of this rare feature of inflammatory bowel disease. 


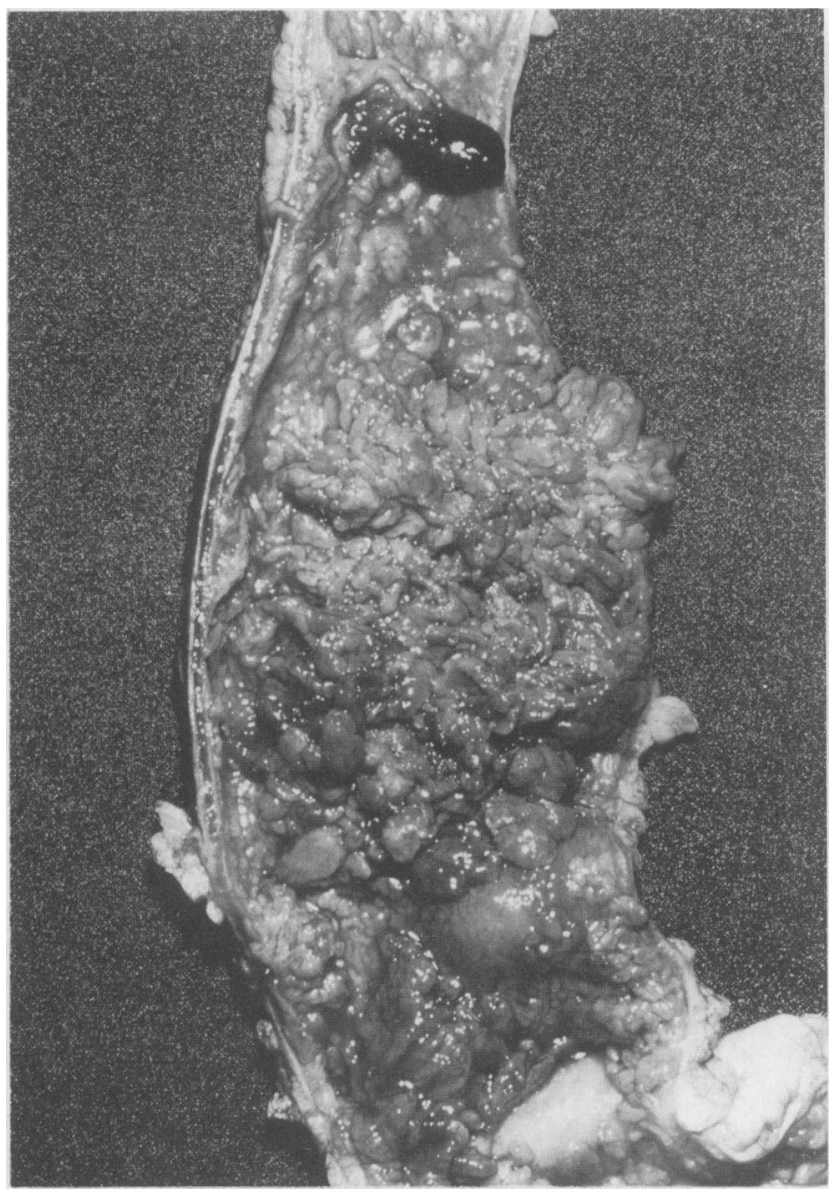

FIG. 3. Pathology specimen of colon showing localized mass of pseudo-polyps.

\section{References}

Bockus, H.L., Roth, J.L.A., Buchman, E. \& KalSER, M. (1958) Ulcerative colitis; classification of types, clinical behaviour, life history and prognosis. In: Modern trends in gastroenterology (Ed by Jones, F.A.), 2nd series, p. 296. Butterworth, London.

DE Dombal, F.T., WatTs, J. MCK., WatKInSON, G. \& Goligher, J.C. (1966) Local complications of ulcerative colitis: stricture, pseudo-polyposis and carcinoma of colon and rectum. British Medical Journal, 1, 1442.

EDWARDS, F.C. \& TRUELOVE, S.C. (1964) The course and prognosis of ulcerative colitis. Gut, 5, 1.

FERRUCI, J.F., JR \& VICKERY, A.L., JR (1972) Case records of the Massachusetts General Hospital. New England Journal of Medicine, 286, 147.
Jalan, K.N., Sircus, W., Walker, R.J. \& MCManus, J.P. (1969) Pseudo-polyposis in ulcerative colitis. Lancet, ii, 555.

JOFFE, N. (1977) Localized giant pseudo-polyposis secondary to ulcerative or granulomatous colitis. Clinical Radiology, 28, 609.

KeatiNG, J.W. \& Mindell, H.J. (1976) Localized giant pseudopolyposis in ulcerative colitis. American Journal of Roentgenology, 126, 1178.

MORSON, B.C. \& PANG, L.S.C. (1967) Rectal biopsy as an aid to cancer control in ulcerative colitis. Gut, 8, 423.

WILLS, J.S. \& HAN, S.S. (1977) Localized giant pseudo-polyposis complicating granulomatous ileocolitis. Radiology, 122, 320. 